\title{
Optimization of Collocated/Noncollocated Sensors and Actuators along with Feedback Gain Using Hybrid Multiobjective Genetic Algorithm-Artificial Neural Network
}

\author{
Deepak Chhabra, ${ }^{1}$ Gian Bhushan, ${ }^{2}$ and Pankaj Chandna ${ }^{2}$ \\ ${ }^{1}$ Department of Mechanical Engineering, University Institute of Engineering \& Technology, Maharshi Dayanand University, \\ Rohtak, Haryana 124001, India \\ ${ }^{2}$ Department of Mechanical Engineering, National Institute of Technology, Kurukshetra, Haryana 136119, India
}

Correspondence should be addressed to Deepak Chhabra; deepak.chhabra@mdurohtak.ac.in

Received 16 November 2013; Accepted 24 December 2013; Published 20 February 2014

Academic Editors: X. Guan, B. Sun, and G. Xie

Copyright (C) 2014 Deepak Chhabra et al. This is an open access article distributed under the Creative Commons Attribution License, which permits unrestricted use, distribution, and reproduction in any medium, provided the original work is properly cited.

A multiobjective optimization procedure is proposed to deal with the optimal number and locations of collocated/noncollocated sensors and actuators and determination of LQR controller gain simultaneously using hybrid multiobjective genetic algorithmartificial neural network (GA-ANN). Multiobjective optimization problem has been formulated using trade-off objective functions ensuring good observability/controllability of the structure while minimizing the spillover effect and maximizing closed loop average damping ratio. Artificial neural networks (ANNs) are used to train the input as varying numbers and placements of sensors and actuators and the outputs are taken as the three objective functions (i.e., controllability, observability, and closed loop average damping ratio), thus forming three ANN models. The trained mathematical models of ANN are fed into the multiobjective GA. The hybrid multiobjective GA-ANN maintains the trade-off among the three objective functions. The ANN3 model is used experimentally to provide the control inputs to the piezoactuators. It is shown that the proposed method is effective in ascertaining the optimal number and placement of actuators and sensors with consideration of controllability, observability, and spillover prevention such that the performance on dynamic responses is also satisfied. It is also observed that damping ratio obtained with hybrid multiobjective GA-ANN and found with ANN experimentally/online holds well in agreement.

\section{Introduction}

The smart structures and materials have been used extensively for active control of sound and vibration, structural health monitoring, shape control, and energy harvesting. Piezoelectric materials have been widely used as sensors and actuators for active vibration control because piezoelectric materials provide inexpensive, reliable, fast response, large operating bandwidth, and low weight, low power consumption while actuating and sensing the vibrations in flexible structures. It is well known that performance of active vibration control depends not only on control law but also on sensor/actuator selection and placement [1]. Misplaced sensors and actuators lead to some problems such as lack of observability and controllability and spillover. The optimal placement of sensors/actuators has been obtained using various objective functions like maximizing degree of controllability, minimizing control effort, minimizing spillover effects, maximizing modal forces applied by piezoelectric actuators and optimizing techniques like genetic algorithm (GA), simulated annealing (SA), multi-objective genetic algorithm (MOGA), sequential best adding (SBA) algorithm, penalty function method, Swarm intelligence algorithm, modified GA (MGA), tabu search method, and so forth [2]. The collocated sensor/actuator locations are preferred over noncollocated as non-collocated sensor/actuator location can lead to instability of the closed loop system. However, the optimal sensor/actuator location, together with the optimal feedback control gain, can make the structural control systems stable [3]. Onoda and Haftka [4] simultaneously 
optimized the design of structure and control, in which the optimal structural stiffness and the collocated sensor/actuator location were obtained by maximizing a performance index. Tzou and $\mathrm{Fu}$ [5] investigated that vibrational modes cannot be controlled using fully laminated piezoelectric sensors and actuators because of the lack of observability and controllability. Therefore, patches of the sensors and actuators were used to control most vibrational modes simultaneously. Locations of both sensors and actuators have been determined with consideration of controllability, observability, and spillover prevention [6]. Bruant and Proslier [7] proposed modified method for placement of sensors/actuators ensuring good observability or good controllability of the structure and considering residual modes to limit the spillover effects. Genetic algorithms (GA) have been efficiently applied to find optimal locations of piezoelectric sensors and actuators on beams, plates and shells [8-12]. Multiobjective GA has been applied for both the placement and the dynamic responses [13]. ANNs have been used extensively for active control of sound and vibration and its mathematical modeling within the last decades [14-19].

In most of the studies described above, the number of the sensors and actuators remains fixed and the placements of sensors/actuators are determined considering degree of controllability/observability and spillover prevention. Further, after placing the sensors and actuators, optimal feedback is determined according to the control law. Non-collocated placement leads to the instability and sometimes even collocated placements of sensors and actuators lead to instability, if optimal/suitable gain is not provided to the multi-input multioutput (MIMO) system. Moreover, if we place the collocated sensor/actuators, then it may or may not ensure both controllability and observability.

In the present work, the positions of non-collocated/collocated sensors and actuators with varying numbers (18) on square cantilever plate have been determined along with optimal gain of LQR controller simultaneously while ensuring good controllability/observability and limiting the spillover effects to control the first six modes of vibration. The three objective functions are used with constraints to find optimal number and placement of sensors and actuators and optimal controller gain to maximize closed loop average damping ratio with hybrid multi-objective GA-ANN. The first objective function is taken as minimization of the performance index given by Hać and Liu [6] which considers the number of modes to be controlled and residual modes to limit the spillover effects. The degree of controllability [7] is taken as inequality constraints to determine the optimal number of actuators required to control specified modes. The second objective function is taken as minimization of performance index corresponding to the observability [6] which considers the number of modes to be observed and residual modes to limit the spillover effects to find optimal locations of sensors. The degree of observability is taken as inequality constraints to determine the optimal number of sensors required to observe specified modes. The third objective function is taken as the optimal controller gain to maximize the closed loop average damping ratio while keeping the actuator voltage below the breakdown voltage and maintaining the system stability.

The inputs to the finite element mathematical model of cantilever plate (MATLAB 2010b) are given as the numbers (1-8) and positions (1-64) of the actuators and sensors and the outputs obtained from the FE model as the values of the three objective functions. The inputs and the outputs of the FE model are the inputs and the targets given to the ANN. The three artificial neural networks have been trained with feed forward back propagation NN. The three trained ANN model is fed to the multiobjective GA and third one used experimentally. The hybrid multi-objective GA-ANN has been successfully used to find the Pareto optimal set. Instead of single solution, the Pareto optimal set provides a number of solutions between trade-off objectives. It is shown that simultaneous optimization gives a number of options to select number and placement of sensors/actuators according to the required dynamic response.

\section{Methodology}

A square cantilever plate is considered with side $L$ and thickness $h$ made up of isotropic elastic material. The plate is bonded with piezoceramic PZT-5A (lead Zirconium Titnate) patches polarized in the thickness direction. The plate is modeled using the finite element method. It is divided into 64 discrete finite elements of length $2 \mathrm{a}$ as shown in Figure 2. The element consists of four nodes $i, j, k$, and $m$ and each node has three degrees of freedom $w_{i}, \theta_{x i}$, and $\theta_{y i}$, and thus, total degree of freedom of smart plate becomes $64 \times 12=243$ as shown in Figure 1.

By defining the local coordinates $\xi=x / a$ and $\eta=$ $y / b$ which are originated at the center of the element, the transverse displacement of the element is interpolated as

$$
w_{i}=\left[N_{i}(\xi, \eta) N_{j}(\xi, \eta) N_{l}(\xi, \eta) N_{m}(\xi, \eta)\right][u]_{e},
$$

where $u_{e}=\left\{w_{i} \theta_{x i} \theta_{y i}, \ldots, w_{m} \theta_{x m} \theta_{y m}\right\}^{T}$,

$$
\begin{array}{r}
N_{k}^{T}(\xi, \eta) \\
\quad=\frac{1}{8}\left[\begin{array}{c}
\left(1+\xi_{k} \xi\right)\left(1+\eta_{k} \eta\right)\left(2+\xi_{k} \xi+\eta_{k} \eta-\xi^{2}-\eta^{2}\right) \\
b\left(1+\xi_{k} \xi\right)\left(\eta_{k}+\eta\right)\left(\eta^{2}-1\right) \\
-a\left(\xi_{k}+\xi\right)\left(\xi^{2}-1\right)\left(1+\eta_{k} \eta\right)
\end{array}\right] \\
k=i, j, l, m .
\end{array}
$$

Ignoring shear deformations in the plate and using Kirchhoff's classical plate theory, strains $\{\varepsilon\}$ developing in the plate can be written as

$$
\{\varepsilon\}=\left\{\frac{\partial u}{\partial x} \frac{\partial v^{\prime}}{\partial y} \frac{\partial v^{\prime}}{\partial x}+\frac{\partial u}{\partial x}\right\}^{T},
$$

where $u=-z(\partial w / \partial x)$ and $v^{\prime}=-z(\partial w / \partial y)$.

After substituting values of " $u$ " and " $v$ " in (3), we get

$$
\{\varepsilon\}_{3 \times 1}=z\left[B_{e}\right]_{3 \times 12}\left\{u_{e}\right\}_{12 \times 1} \text {, }
$$




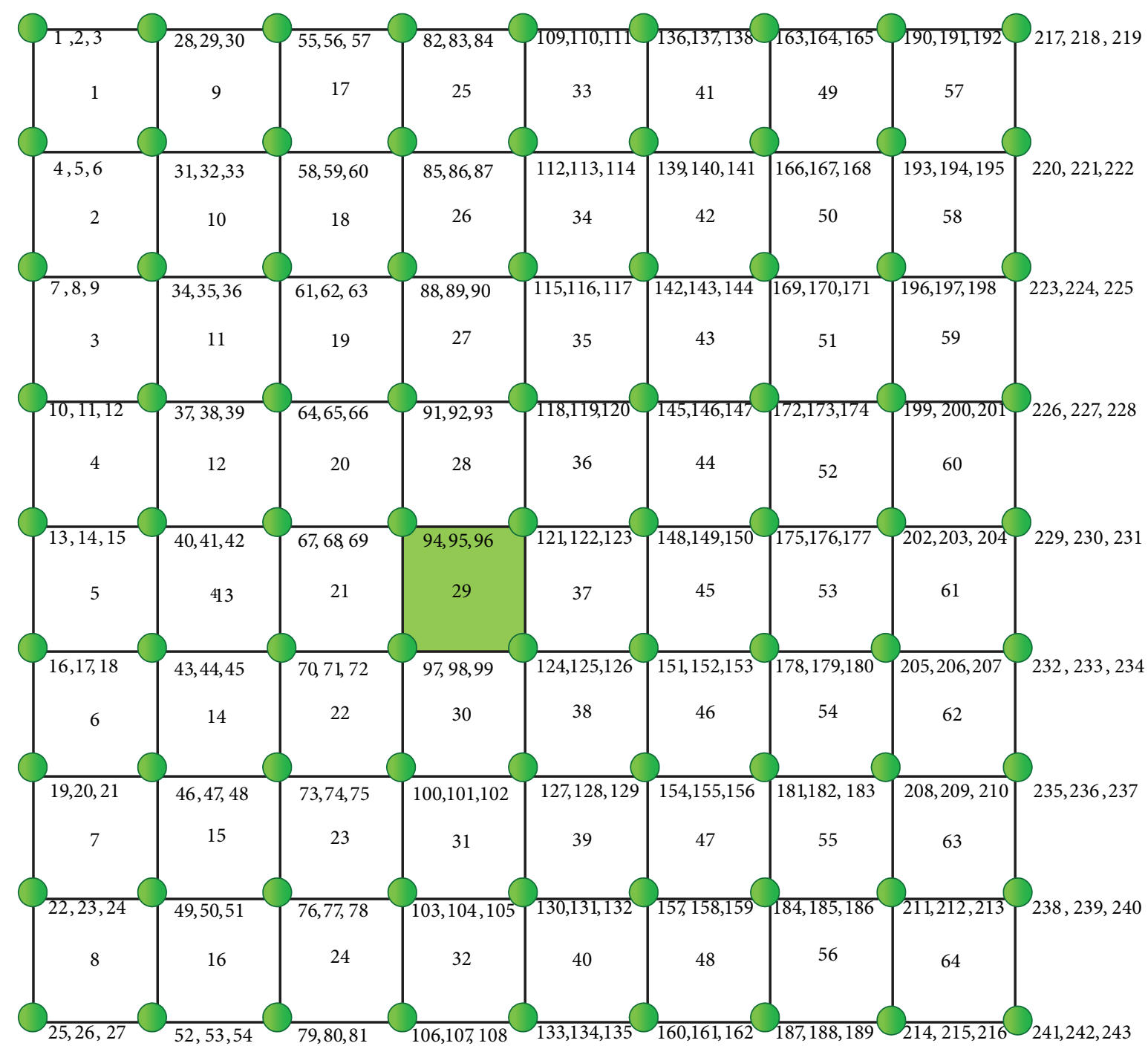

FIGURE 1: Degrees of freedom of finite element smart plate.

where $\left[B_{e}\right]_{3 \times 12}=\left[-\left(z \partial^{2} / \partial x^{2}\right)-\left(z \partial^{2} / \partial y^{2}\right)-\left(2 z \partial^{2} /\right.\right.$ $\partial x \partial y)]_{3 \times 1}^{T}[N]_{1 \times 12}$.

Kinetic energy $\left(T_{e}\right)$ of a finite element is

$$
T_{e}=\frac{1}{2} \int_{s} \rho_{s} \dot{w}^{2} d \tau+\frac{1}{2} \int_{p} \rho_{p} \dot{w}^{2} d \tau .
$$

Potential energy $\left(V_{e}\right)$ of a finite element is

$$
V_{e}=\frac{1}{2} \int_{s}\{\varepsilon\}^{T}\{\sigma\} d \tau+\frac{1}{2} \int_{v}\{\varepsilon\}^{T}\{\sigma\} d \tau .
$$

Using Hamilton's principle, the equation of motion of one finite element with piezo-patches actuators and sensors are symmetrically bonded to the top and the bottom surfaces contains two variables namely " $\left\{u_{e}\right\}$ " and " $v$ ". Taking variation with respect to $\left\{u_{e}\right\}$, we get

$$
\left(\left[m_{s}^{e}\right]+\left[m_{P}^{e}\right]\right)\left\{\ddot{u}_{e}\right\}+\left(\left[k_{s}^{e}\right]+\left[k_{P}^{e}\right]\right)\left\{u_{e}\right\}+\left[k_{u v}^{e}\right] v=\left\{F_{s}^{e}\right\},
$$

and taking variation with respect to " $v$ ", we get

$$
\begin{aligned}
& {\left[k_{v u}^{e}\right]\left\{u_{e}\right\}-\left[k_{v v}^{e}\right] v=0} \\
& v=\left[k_{v v}^{e}\right]^{-1}\left[k_{v u}^{e}\right]\left\{u_{e}\right\},
\end{aligned}
$$

where

$$
\begin{gathered}
{\left[m_{s}^{e}\right]=\rho a b h \iint_{-1}^{1}[N]^{T}[N] d \xi d \eta,} \\
{\left[m_{P}^{e}\right]=\rho_{p e} a b h_{p e} \iint_{-1}^{1}[N]^{T}[N] d \xi d \eta,} \\
{\left[k_{s}^{e}\right]=\left(\frac{a b h^{3}}{12}\right) \iint_{-1}^{1}[B e]^{T}[C]\{B e] d \xi d \eta,} \\
{\left[k_{p}^{e}\right]=\left(z^{2}\right) \iint_{-1}^{1}[B e]^{T}\left[C_{p}\right]\{B e] d \xi d \eta,}
\end{gathered}
$$




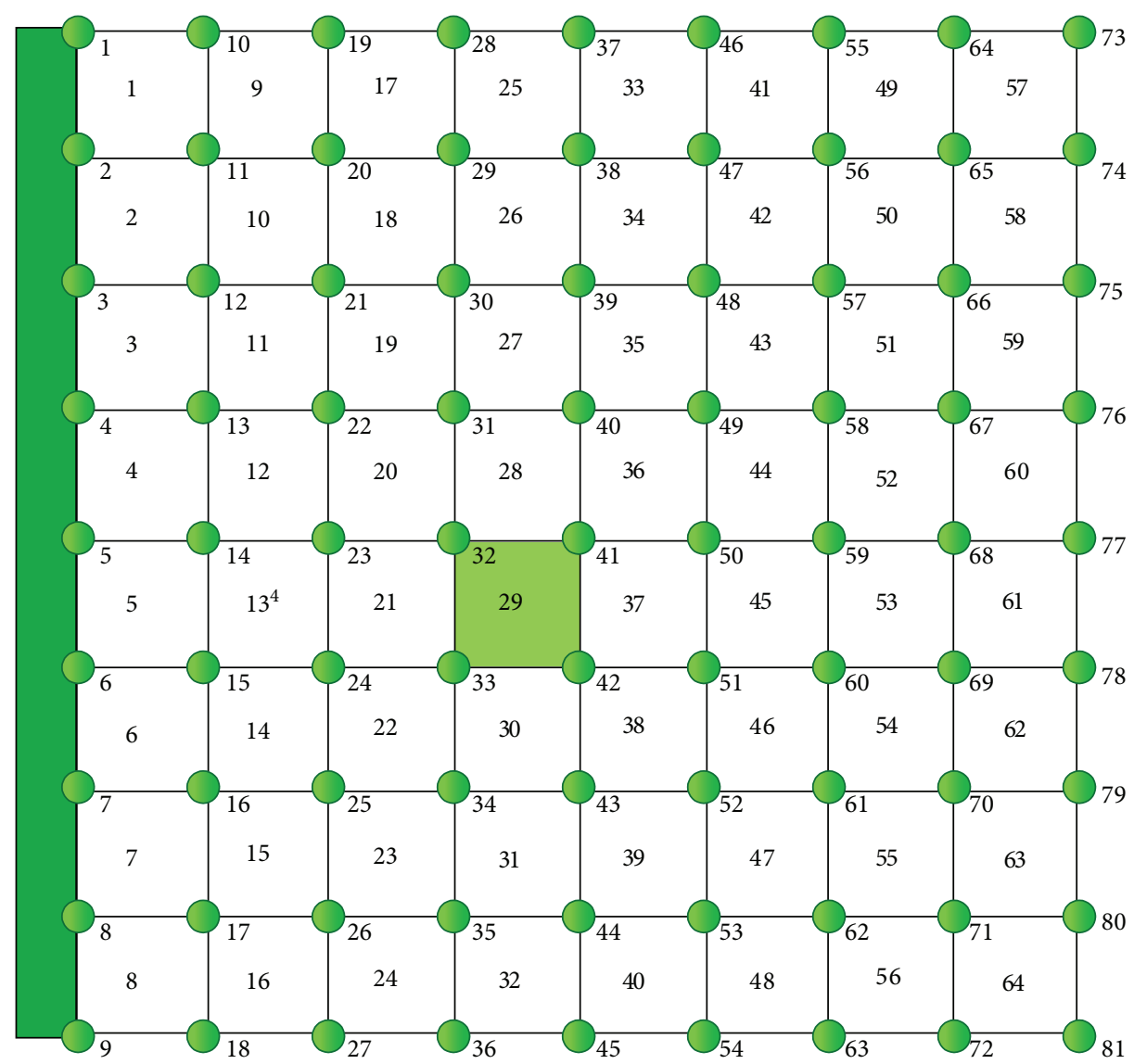

FIGURE 2: Finite element smart cantilever plate with node numbering.

$$
\begin{gathered}
{\left[k_{u v}^{e}\right]=a b h_{p e}\left(h+\frac{h_{p e}}{2}\right) \iint_{-1}^{1}[B e]^{T}[e]\left\{B_{v}\right] d \xi d \eta,} \\
{\left[k_{v u}^{e}\right]=a b h_{p e}\left(h+\frac{h_{p e}}{2}\right) \iint_{-1}^{1}\left[B_{v}\right]^{T}[e]^{T}\{B e] d \xi d \eta,} \\
{\left[k_{v v}^{e}\right]=4 a b h_{p e} \iint_{-1}^{1}\left[B_{v}\right]^{T}[X]\left\{B_{v}\right] d \xi d \eta,} \\
\left\{F_{s}^{e}\right\}=\int\left\{f_{s}^{e}\right\} d s,
\end{gathered}
$$$$
[C p]=\left[\begin{array}{ccc}
1 & \nu^{\prime} & 0 \\
\nu^{\prime} & 1 & 0 \\
0 & 0 & \frac{1-\nu^{\prime}}{2}
\end{array}\right], \quad[C]=\left[\begin{array}{ccc}
1 & v & 0 \\
\nu & 1 & 0 \\
0 & 0 & \frac{1-\nu}{2}
\end{array}\right] \text {, }
$$$$
\left[B_{v}\right]=\left[\begin{array}{c}
0 \\
0 \\
1 \\
\hline h_{p e}
\end{array}\right] \text {, }
$$$$
[e]=\left[\begin{array}{ccc}
0 & 0 & 0 \\
0 & 0 & 0 \\
e_{31} & e_{31} & 0
\end{array}\right], \quad[X]=\left[\begin{array}{ccc}
X_{11} & 0 & 0 \\
0 & X_{11} & 0 \\
0 & 0 & X_{33}
\end{array}\right] \text {. }
$$

The mass and stiffness matrices can be easily computed via numerical integration by taking the value of abscissa and weight coefficients of the Gaussian quadrature formula as $a=\left[\begin{array}{ll}0.8611363116 & -0.86113631160 .3399810436-0.33998104\end{array}\right.$ $36]$ and $w=\left[\begin{array}{lll}0.3478548451 & 0.34785484510 .6521451549 & 0.65\end{array}\right.$ 21451549] to solve the inner integral [20]. The piezoelectric mass, stiffness, and electromechanical coupling have been added with plate/substrate element at the positions of piezoelectric patches. Using an assembly procedure, the global equation of motion of the smart piezoplate structure becomes

$$
[M]_{243 \times 243}\{\ddot{x}\}_{243 \times 1}+[K]_{243 \times 243}\{x\}_{243 \times 1}=\{F\}_{243 \times 1} .
$$

Due to boundary conditions, left edge of the plate has been fixed as shown in Figure 2. The degree of freedom reduces to $(243-27=216)$ and equation of motion of smart plate becomes

$$
[M]_{216 \times 216}\{\ddot{x}\}_{216 \times 1}+[K]_{216 \times 216}\{x\}_{216 \times 1}=\{F\}_{216 \times 1} .
$$

The final equation of motion of the smart piezo plate structure with damping becomes

$$
\begin{gathered}
{[M]_{216 \times 216}\{\ddot{x}\}_{216 \times 1}+\left[D_{p}\right]_{216 \times 216}\{\ddot{x}\}_{216 \times 1}} \\
+[K]_{216 \times 216}\{x\}_{216 \times 1}=\{F\}_{216 \times 1},
\end{gathered}
$$

(9) where $D_{p}=\alpha[M]+\beta[K]$. 
Equation (12) represents a coupled system of equations. Analysis of such a system becomes easier if these coupled equations are converted into uncoupled equations by using the following transformation:

$$
\{x\}_{n_{d} x 1}=[U]_{n_{d} x n}\{\eta\}_{n x 1} .
$$

Restricting the analysis to the first six modes only, the uncoupled equations of motion are given as

$$
\ddot{\eta}_{i}(t)+2 \zeta_{i} \omega_{i} \dot{\eta}_{i}(t)+\omega_{i}^{2} \eta_{i}(t)=f_{i}=\sum_{n=1}^{N_{a}} B_{i} \emptyset_{a_{n}}(t) .
$$

$i$ is the mode number and $B_{i}$ is the $i$ th modal component of control force due to electric potential $\emptyset_{a_{n}}$ applied to the $n$th actuator which depends on not only the modal shape functions but also the locations and sizes of the actuator elements. Equation (12) can be transformed in to state space equations as equations as

$$
\{\dot{x}\}=[A]\{x\}+\left[B_{c}\right]\{u\},
$$

where $A=\left[\begin{array}{cc}0_{n x n} & I_{n x n} \\ -M^{-1} K & -M^{-1} D_{p}\end{array}\right]_{2 n x 2 n}, u=\left[\emptyset_{a 1} \emptyset_{a 2}, \ldots, \emptyset_{a_{n}}\right]^{T}$. $I$ and 0 stand for the identity and null matrices, respectively

$$
\{y\}=[C]\{x\} .
$$

where $y=\left[q_{1}, \ldots, q_{n s}\right]^{T}$, and $C=\left[\begin{array}{ccc}0 & \ldots & 0 \\ C_{11} & \ldots & C_{n_{s} 1} \\ \vdots & \vdots & \vdots \\ 0 & \vdots & 0 \\ C_{n 1} & \ldots & C_{n_{s} N}\end{array}\right]$.

2.1. Optimization Problem Formulation for the Optimal Locations and Number of Actuators and Sensors. The minimum energy control for the regulation of the structural vibration has been considered, to facilitate the performance criterion for sensors and actuators locations [6]. The initial condition of the state vector $x$ in (15) is assumed to be $x_{0}$. The minimal energy control problem to regulate the state $x$ to $x_{0}$ within given time $t_{1}$ is given as follows:

$$
\operatorname{minimize} I(u)=\int_{0}^{t_{1}} u^{T}(t) u(t) d t
$$

subject to $x(0)=x_{0}, x\left(t_{1}\right)=0$, and (15).

The solution of (17) is

$$
I_{1}=\left(\begin{array}{ll}
e^{A t_{1}} & x_{0}
\end{array}\right)^{T} W^{-1}\left(t_{1}\right)\left(\begin{array}{ll}
e^{A t_{1}} & x_{0}
\end{array}\right)
$$

where $W(t)$ is called the controllability Gramian, defined as

$$
W(t)=\int_{0}^{t} e^{A \tau} B B^{T} e^{A^{T} \tau} d \tau .
$$

$W(t)$ depends on time $t_{1}$. Hać and Liu [6] proposed an objective function where, instead of using $W(T)$, steady state controllability Gramian $W_{c}$ can be considered to eliminate the dependency of the solution $T$. The objective function for actuator location is to minimize the performance index (PI):

$$
\begin{aligned}
& \mathrm{PI}=\left(\sum_{j=1}^{2 n} \lambda_{j}\right)^{2 n} \sqrt{\prod_{j=1}^{2 n} \lambda_{j}}-\gamma\left(\sum_{k=2 n+1}^{2 N} \lambda_{k}\right)^{2(N-n)} \sqrt{\prod_{k=2 n+1}^{2 N} \lambda_{k}} \\
& \text { subject to Code } g_{i}=\frac{\sum_{j=1}^{N_{a}} b_{i j}^{2}}{\max b_{i 1}^{2}}>1 .
\end{aligned}
$$

$n$ are the modes to be controlled, $N$ is the total number of the modes of model including residual modes, $\lambda_{j}$ is the eigenvalue of the steady state controllability Gramian related to the corresponding mode to be controlled, $\lambda_{k}$ is the eigenvalue of the steady state controllability Gramian related to the corresponding residual mode, and $\gamma$ is the weighting constant. Code $g_{i}$ is the degree of controllability of $i$ th mode which is equal to the energy transmitted from the actuators to the structure for the $i$ th mode divided by the maximal value energy obtained if the $i$ th mode is optimally controlled by one actuator. Thus, the optimization problem consists in finding the number of actuators $n_{a}$ and the placement of the actuators, which gives the optimal number of actuators and minimizes the function PI to control the desired modes subject to Code $g_{i}>1$ [7].

The performance criterion for the sensor numbers and locations can be obtained using a similar approach. This method ensures good controllability and observability considering the effect of residual modes.

2.2. GA-LQR Optimal Control. LQR optimal control theory is used to determine the active control gain. The following quadratic cost function is minimized:

$$
j=\frac{1}{2} \int_{0}^{\infty}\left(\{x\}^{T}\{x\}^{T}[Q]\{x\}+\{u\}^{T}[R]\{u\}\right) d t .
$$

$[Q]$, of dimension $(2 n \times 2 n)$, and $[R]$, of dimension $\left(n_{a} x n_{a}\right)$, control the value of the performance index. They are the main design parameters. $J$ represents the weighted sum of energy of the state and control.

Assuming full state feedback, the control law is given by

$$
\{u\}=-[K]\{x\}
$$

with constant control gain

$$
[K]=[R]^{-1}[B]^{T}[S] .
$$

Matrix $S$ can be obtained by the solution of the Riccati equation, given by

$$
[A]^{T}[S]+[S][A]+[Q]-[S][B][R]^{-1}[B]^{T}[S]=0 .
$$

Solution of the reduced Riccati equation (24) gives the value of matrix [S]; if matrix [S] is positive definite, then the system is stable and the closed loop matrix $[A]-[B][K]$ is stable. The feedback control gain matrix can be obtained after substitution of $[S]$ in (23). 
$Q$ and $R$ matrices can be determined by considering weighted energy of the system $[21,22]$ :

$$
\begin{gathered}
{[Q]=\left[\begin{array}{cc}
\alpha_{1} U^{T} K t U & 0 \\
0 & \beta_{1} U^{T} M t U
\end{array}\right],} \\
{[R]=\gamma_{1}[\widehat{R}],}
\end{gathered}
$$

where $\alpha_{1}, \beta_{1}$, and $\gamma_{1}$ are the coefficients associated with total kinetic energy, strain energy and input energy respectively, $[\widehat{R}]$ is the dielectric coupling matrix of the actuators and $M t$ and $K t$ are the total mass and stiffness (sensors, actuators, and substrate) of the system. The weighing matrices have been determined by varying the value of $\alpha_{1}, \beta_{1}$, and $\gamma_{1}$ within $0<\alpha_{1} \leq 200,0<\beta_{1} \leq 200$, and $0<\gamma_{1} \leq 2$, respectively. Closed loop average damping ratio has been considered as fitness function, while keeping the actuators voltage below the breakdown voltage and maintaining the stability of the system. Closed loop average damping ratio is calculated by taking the average of damping ratio of first six modes by using following equation:

$$
\xi_{d c}=\left\{\sum_{c=1}^{n} \frac{1}{n}\left(\frac{1}{\sqrt{1+\left(4 \pi^{2} / g^{2}\right)}}\right)\right\} .
$$

The closed loop average damping ratio has been maximized to determine the parameters of weighing matrices using GA, thus forming GA-LQR. The fitness function for the present case has been taken as

$$
\xi_{d c}=\operatorname{maximize}\left\{\sum_{c=1}^{n} \frac{1}{n}\left(\frac{1}{\sqrt{1+\left(4 \pi^{2} / g^{2}\right)}}\right)\right\}
$$

subjected to $\phi_{i}<\phi_{\max }, i=1, \ldots, n_{a}$, and real eigenvalues $\left(A-B R^{-1} B^{T} S\right)<0$.

\section{Artificial Neural Network Modeling}

The inputs, that is, positions of piezoelectric sensors/actuators and parameters related to weighing matrices, are fed into the finite element model described above and outputs obtained are the three objective functions. Firstly, the eight piezopatches positions are given to the model and outputs are obtained. Then by reducing one sensor/actuator each time, outputs are obtained. A total of 256 numerical simulations are performed based on 2-level factorial design where each factor is varied over two levels. The input matrix is taken of the order of $19 \times 256$ (inputs $\times$ simulations) and output matrix is obtained of the order of $1 \times 256$ (first objective functions values $\times$ simulations), thus forming the first neural network (Figure 3(a)). The neural feed forward back propagation network has been used to train the model. The tansig and purelin transfer functions and trainlm algorithm are used. The graph between outputs and the targets has been obtained as shown in Figure 3(b). Similarly, two NN have been designed by taking the second and third objective functions value in output matrix while the input matrix remains the same.

\section{Experimental Setup}

The first six modal frequencies of cantilever plate obtained with finite element code using MATLAB have been compared with the experimental results obtained using National Instruments (NI) cRIO 9014 real time controller with compact RIO 9103 reconfigurable chassis, along with NI 9234 DAQ for piezosensor. The cRIO 9263 analog output module along with amplifier has been used for piezoactuation. A PZT-5A square patch is used as sensor and actuator. The material properties and dimensions of plate and piezo-patches are given in Table 2. Both piezoceramic patches have been fine soldered with connecting wires on top and bottom surfaces. The epoxy adhesive has been used for bonding the piezopatches with plate perfectly. The signal from the sensor is fed into the NI 9234 DAQ through BNC connector. The four input channels simultaneously digitize signals at rates up to $51.2 \mathrm{kHz}$ per channel with built-in filters that automatically adjust the sampling rate. The NI 9234 DAQ card and NI 9263 analog output module have been mounted on the cRIO 9103 reconfigurable chassis along with cRIO 9014 real time controller. The real time controller is connected to the PC via Ethernet cable. The entire experimental set-up is shown in Figures 4(a) and 4(b). Figure 5 shows the schematic diagram of the experimental set up. A.VI (virtual instrumentation) program is written in "LabView 2012 Sp1" and burnt in cRIO 9014 real time controller via Ethernet cable and run there. The cantilever plate was displaced by instrumented hammer and the transient responses were recorded in LabView. With the help of FFT (fast Fourier transform) analyser the first six modes were obtained. The comparison of natural frequencies is as shown in Table 2.

It is observed from Table 2 that the results obtained from the present finite element code are in close agreement with experimental results.

\section{Optimal Placement of Sensors-Actuators as well as Minimization of Dynamic Responses Using Multiobjective Genetic Algorithm-Artificial Neural Network}

Instead of solving the optimization problem of sensors/actuators number and placement with dynamic responses as sequential optimization, the simultaneous optimization using MOGA gives good physical meaning. A solution is extreme with respect to one objective requiring a compromise in other objectives [23]. This prohibits one from choosing a solution which is optimal with respect to only one objective. Moreover, a number of solutions have been obtained with multiobjective GA which gives a number of options to the designer to choose according to the requirement/applications.

To find the number and optimal locations of noncollocated/collocated sensors and actuators while ensuring good observability/controllability and limiting the spillover prevention, as well as determining feedback gain of LQR controller for maximizing the closed loop average damping ratio while keeping the actuators voltage below the breakdown voltage and considering the stability of the closed 
TABLE 1: Material properties and dimensions of smart plate.

\begin{tabular}{lcc}
\hline Physical parameters & Plate & Piezoelectric sensor/actuator (PZT-5A) \\
\hline Length $(\mathrm{m})$ & 0.16 & 0.02 \\
Breath $(\mathrm{m})$ & 0.16 & 0.02 \\
Thickness $(\mathrm{m})$ & $0.6 e-3$ & $0.9 e-3$ \\
Elastic modulus $(\mathrm{Pa})$ & $2.07 e 11$ & $6.3 e 10$ \\
Density $\left(\mathrm{Kg} / \mathrm{m}^{3}\right)$ & 7800 & 7500 \\
Poisson's ratio & 0.3 & 0.3 \\
Piezodielectric constant $X_{11}(\mathrm{~F} / \mathrm{m})$ & - & $2.84 e-8$ \\
Piezodielectric constant $X_{33}(\mathrm{~F} / \mathrm{m})$ & - & $2.84 e-8$ \\
Piezoelectric constant $e_{31}=e_{32}(\mathrm{~V}-\mathrm{m} / \mathrm{N})$ & - & -24.48 \\
\hline
\end{tabular}

TABLE 2: Natural frequencies of cantilever plate.

\begin{tabular}{lcc}
\hline Mode number & $\begin{array}{c}\text { Present FEM code } \\
(\mathrm{Hz})\end{array}$ & $\begin{array}{c}\text { Experimental } \\
\text { results }(\mathrm{Hz})\end{array}$ \\
\hline 1 & 21.5828016898773 & 20 \\
2 & 53.2315486564473 & 52 \\
3 & 132.455872576241 & 131 \\
4 & 158.866478915661 & 157 \\
5 & 196.623436873934 & 195 \\
6 & 324.059441026423 & 325 \\
\hline
\end{tabular}

loop control system simultaneously, trained ANN models are fed into multiobjective genetic algorithm. The numbers of variables are taken as nineteen. The eight variables are taken for the positions of the sensors and eight for the actuators and three for the determination of the weighing matrices of the LQR controller. The absolute values have been taken for sensors and actuators locations and decimal values for the parameters of weighing matrices within the boundaries. The initial population is generated randomly of variable length for sensors and actuators locations with creation function which vary according to the number of available positions in addition to zero position. Zero position means that no sensors/actuators are available. The varying length also should not contain all zeros. Single point crossover has been used while ensuring that no two individuals of generated offspring allowed have the same value (position) in given domain. Similar to single point crossover, uniform mutation with constraint has been used. The outline of multiobjective optimization problem using multi-objective GA-ANN is as shown in Figure 6.

\section{Results and Discussions}

Kirchhoff's cantilever isotropic square plate of size $160 \times$ $160 \times 0.6 \mathrm{~mm}$ has been discretized into $8 \times 8$ quadrangular elements, each element with 4 nodes and each node having 3 degrees of freedom, that is, $w_{z i}, \theta_{x i}$, and $\theta_{y i}$. The square piezoelectric patches of size $20 \times 20 \times 0.9 \mathrm{~mm}$ as sensors/actuators are bonded to the cantilever plate. The size of each of the actuators/sensors is equal to the size of one element of the plate. The piezoelectric mass, stiffness, and electromechanical coupling have been added to the plate element at the positions of piezoelectric patches. The material properties and the dimensions of the smart plate with piezoelectric actuators are given in Table 1. In the FE model of smart plate, the first objective function is minimization of performance index for the placement of actuators [6] which considers the effect of modes to be controlled as well as the residual modes and limits the spillover effects. The degree of controllability has been taken as constraint for determining the optimal number of actuators to control the first six modes of vibration of cantilever plate and residual modes are taken as four. The penalty approach has been applied to implement/calculate the fitness function with constraints. A penalty has been given to the fitness function, if the degree of controllability condition is not satisfied. The piezoelectric actuators mass, stiffness, and electromechanical coupling have been added to the plate element at the positions of piezoelectric actuator in the first objective function. Similarly, the second objective function with constraint is also minimization of performance index to determine the optimal number and location of sensors considering degree of observability and spillover prevention. The piezoelectric sensors mass, stiffness, and electromechanical coupling have been added to the plate element at the positions of piezoelectric sensors. The third objective function has been taken as optimal controller gain for the maximization of closed loop average damping ratio by taking breakdown voltage and stability as constraints, thus forming GA-LQR. In this fitness, the sensors and actuators mass, stiffness, and electromechanical coupling have been considered by adding the system matrices together and taking the output matrix of sensors and input matrix of actuators. The numbers of piezoelectric sensors/actuators have been varied from 1 to 8 . The inputs given to the finite element model of smart cantilever plate are positions and numbers of sensors/actuators and parameters corresponding to the weighing matrices of LQR controller. The outputs obtained from the FE model are the PIc, PIo, and optimal controller gain as described in Sections 2.1 and 2.2.

The inputs given to the FE model and outputs obtained are used to train the ANN. The three ANN mathematical models have been trained. The first ANN1 is the model trained between 19 variable inputs and one output, that is, PIo. The second ANN2 model is trained with 19 variables as input matrix $(19 \times 256)$ and output matrix, that is, PIc $(1 \times 256)$. 


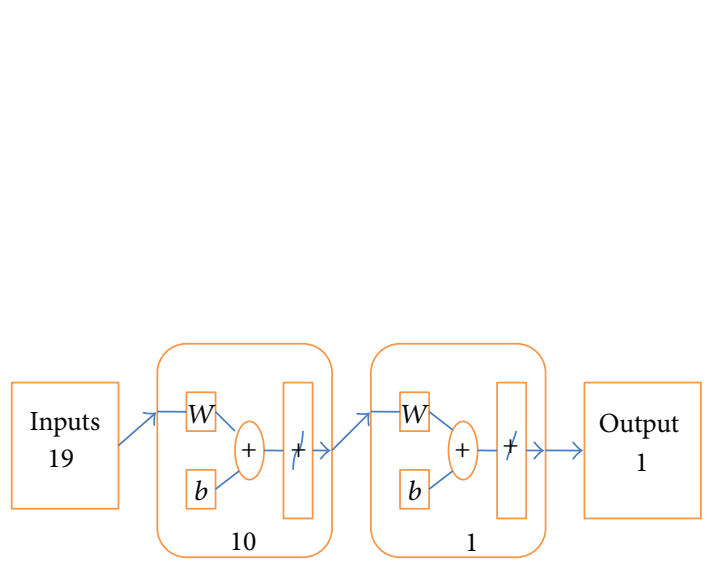

(a)

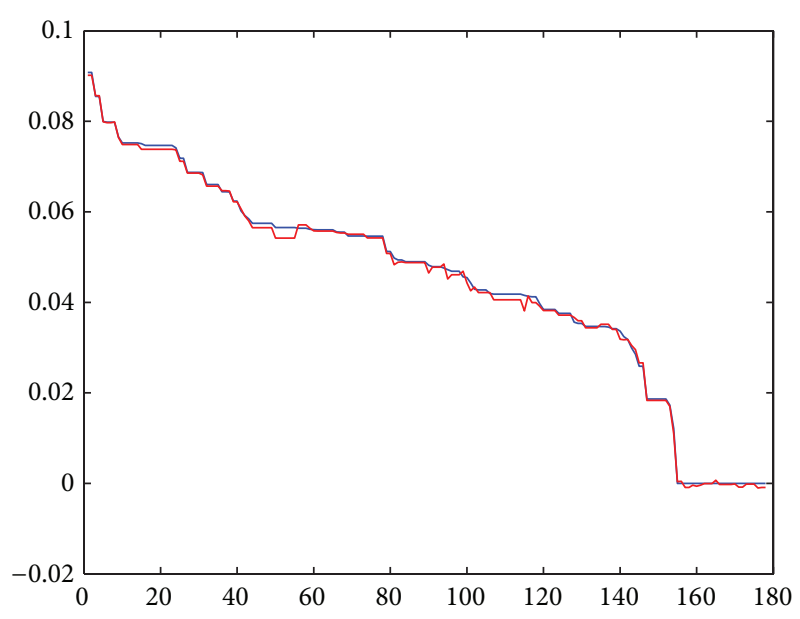

(b)

FIgURE 3: (a) ANN1 model. (b) The graph between outputs and targets.

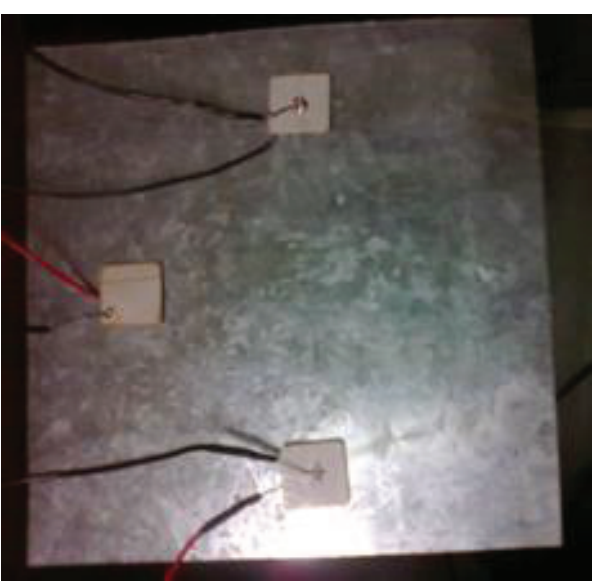

(a)

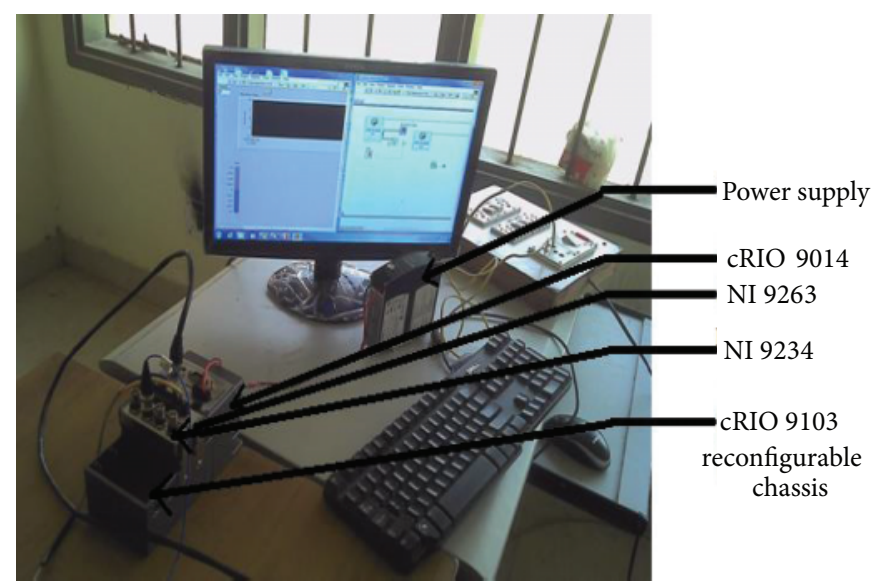

(b)

FIgURE 4: (a) Cantilever plate with the collocated PZT-5A sensor-actuator pair. (b) Experimental setup.

The third ANN3 model is trained with 19 variables as input matrix $(19 \times 256)$ and output as optimal controller gain/control inputs to the actuators. The three trained ANN models are used in multiobjective genetic algorithm,thus forming hybrid multiobjective GA-ANN.

The number of the piezoelectric sensors/actuators has been varied by altering the length (1-8) of chromosome generated using creation function in MOGA. Simultaneous optimization problem has been formulated by taking three ANN models as objective functions. The number of actuators and sensors with positions along with closed loop average damping ratio achieved (in decreasing order) is shown in Table 3. The numbers of solutions are the Pareto optimal set. All of the solutions obtained from the Pareto optimal set have observability/controllability index greater than one to suppress the first six modes of vibrations for cantilever plate. The zero values in Table 3 show that there are no sensors/actuators present there. The first three columns of Table 3 show the parameters associated with weighing matrices to determine the LQR optimal gain. PIc and PIo are the values of the performance index related to the controllability and observability, respectively, which are to be minimized. The results obtained with the Pareto optimal set are very interesting and useful and maintain trade-off among three objective functions.

Some solutions give the maximum damping ratio; others give minimum performance index for controllability/observability. The first solution shows that maximum closed loop average damping ratio can be achieved (0.0907) by using eight non-collocated actuators and sensors at the positions shown in Table 3. Solution number 2 shows that seven actuators and six sensors are used to achieve closed loop average damping ratio of 0.0855 . Here two sensors and actuators are at collocated locations (28 and 55). Solution number 5 shows that the damping ratio achieved is 0.066 with eight actuators and sensors and PIc is minimum among the optimal set. The four sensors and actuators are at collocated location in solution number 11 and the closed loop average 


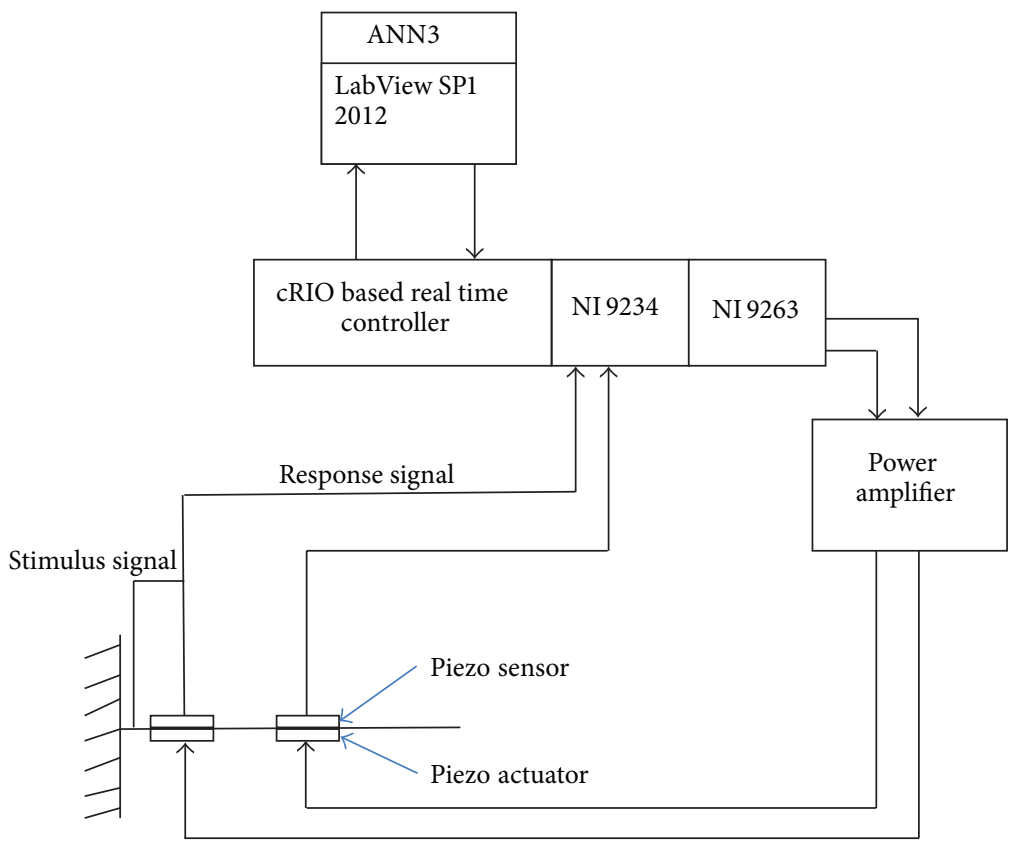

FIGURE 5: Block diagram of experimental setup.

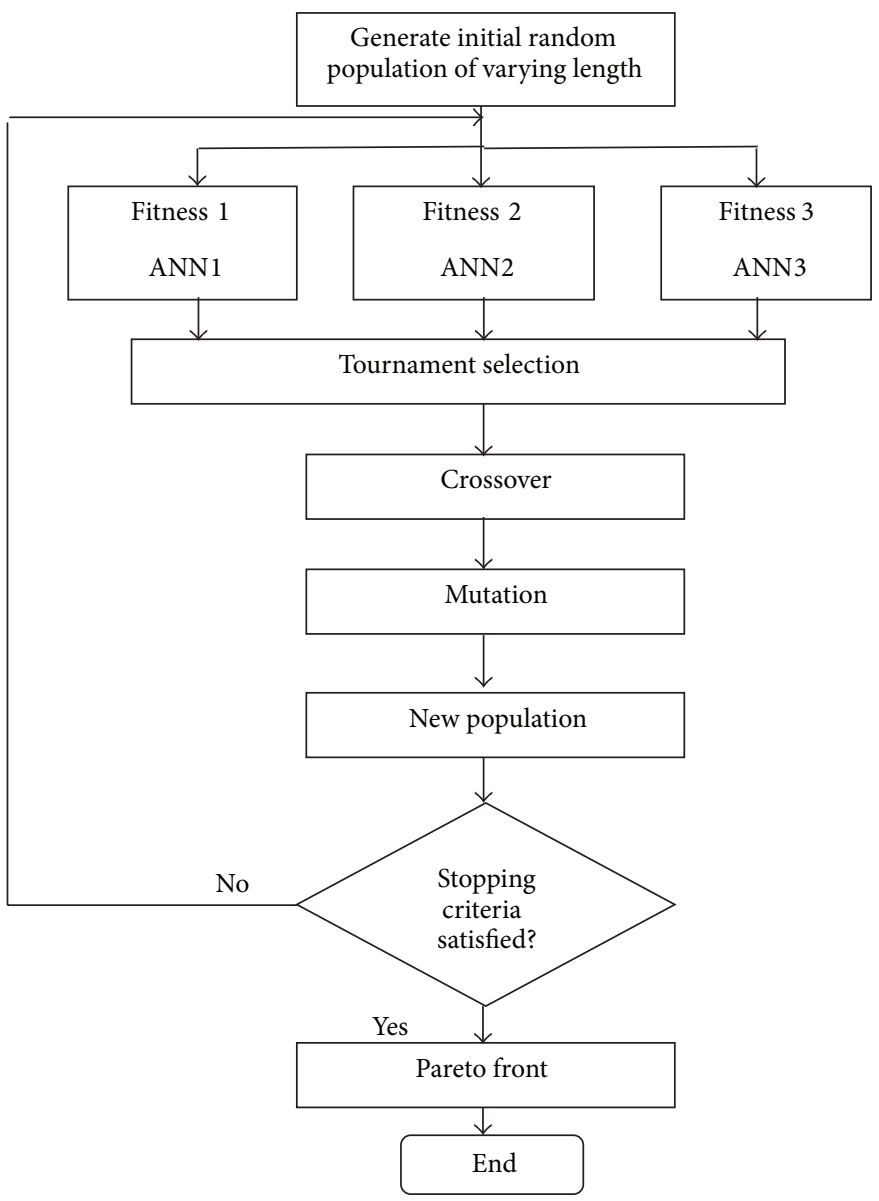

FIGURE 6: Multiobjective optimization using multiobjective GA-ANN. 


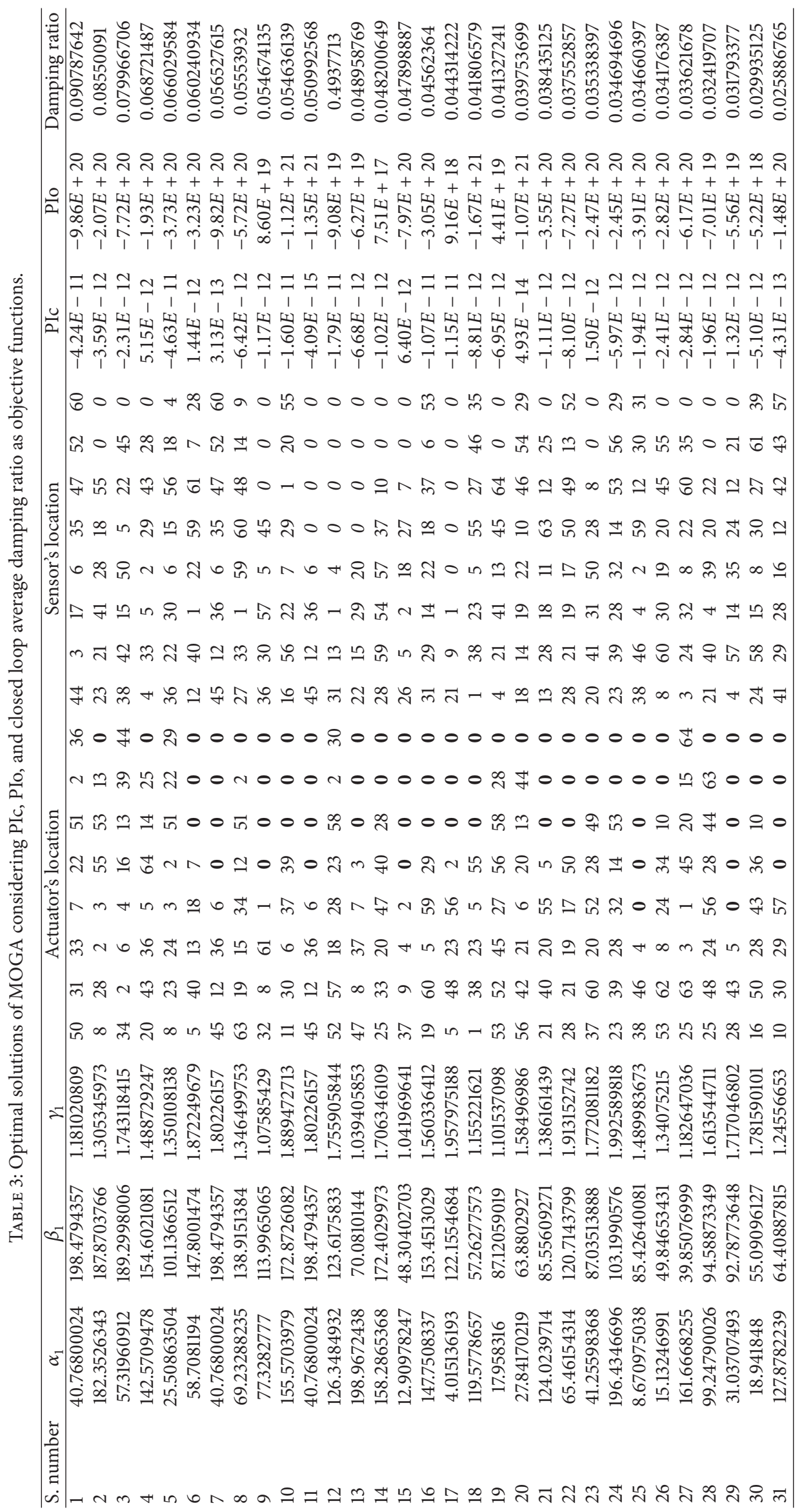


damping ratio achieved is 0.0489 . The PIo is minimum for the solution number 14 with six non-collocated sensors and actuators. Solution number 17 shows that the minimum 3 sensors are required to observe the first six modes of vibration in the present case while solution number 25 shows that minimum three numbers of actuators are required to control the first six modes of vibration.

\section{Conclusions}

Multi-objective optimization has been successfully implemented to find the optimal number and locations of both sensors and actuators on cantilever plate with consideration of controllability, observability, and spillover prevention to control specified six modes of vibrations using multi-objective GA-ANN. The three ANN mathematical models are trained successfully with correlation between targets and outputs, $R=0.99$. The ANN models are used as fitness functions in MOGA. The chromosome of varying length has been successfully generated in MOGA. The collocated and noncollocated sensors/actuators positions have been obtained in the Pareto optimal set with the present approach. Significant vibration reduction for the first six modes (controlled modes) has been observed using simultaneous optimal number and placement of collocated/non-collocated sensors and actuators along with LQR optimal control. Non-collocated sensors/actuators positions also stabilize a control system maintaining asymptotic stability. It is observed that, instead of using six sensors/actuators, only three sensors/actuators optimally located are required to suppress the first six modes of vibrations in the studied application. The third ANN model has been used experimentally and provides optimal controller gain to the piezoactuators. It is also investigated that damping ratio achieved with numerical simulations and experimentally holds well in agreement.

\section{Nomenclature}

$\begin{array}{ll}2 a: & \text { Length of finite element } \\ 2 b: & \text { Breadth of finite element } \\ D_{p}: & \text { Damping coefficient } \\ K_{u u}^{e}: & \text { Elastic stiffness matrix } \\ K_{a a}^{e}: & \text { Structural dielectric stiffness matrix for } \\ & \text { actuator } \\ K_{u a}^{e}: & \text { Piezoelectric coupling matrix for } \\ F_{u}: & \text { actuator } \\ F_{\emptyset_{a}}: & \text { Applied mechanical load vector } \\ \alpha, \beta: & \text { Electrical charge vector } \\ v: & \text { Damping constants } \\ \emptyset_{a_{n}}: & \text { Poisson's ratio } \\ \eta_{i}, \dot{\eta}_{i}, \text { and } \ddot{\eta}_{i}: & \text { Control voltage of } n \text { thodal displacement, velocity, and } \\ \omega_{i}: & \text { acceleration, respectively } \\ \zeta_{i}: & \text { Natural frequency of the } i \text { th mode } \\ {[A]:} & \text { Damping ratio of the } i \text { th mode } \\ {[B]:} & \text { System matrix }\end{array}$

\begin{tabular}{|c|c|}
\hline$[C]:$ & Sensor's output matrix \\
\hline$n_{a}:$ & Numbers of piezo-patches as actuators \\
\hline$\Omega:$ & Objective function \\
\hline$X_{11}, X_{33}:$ & Piezodielectric constants \\
\hline$e_{31}, e_{32}:$ & Piezoelectric constant \\
\hline$\left[m^{e}\right]:$ & Mass matrix of finite element of plate \\
\hline$\left[m^{p}\right]:$ & $\begin{array}{l}\text { Mass matrix of finite element of } \\
\text { piezo-patch }\end{array}$ \\
\hline$D:$ & Bending stiffness of the plate \\
\hline$w_{i}:$ & Transverse displacement at the node $i$ \\
\hline$\theta_{x i}:$ & Rotation about the $x$-axis \\
\hline$\theta_{y i}:$ & Rotation about $y$-axis \\
\hline$\alpha_{1}:$ & $\begin{array}{l}\text { Coefficients associated with total kinetic } \\
\text { energy }\end{array}$ \\
\hline$\beta_{1}:$ & $\begin{array}{l}\text { Coefficients associated with strain } \\
\text { energy }\end{array}$ \\
\hline$\gamma_{1}:$ & $\begin{array}{l}\text { Coefficients associated with input } \\
\text { energy }\end{array}$ \\
\hline$[\widehat{R}]:$ & $\begin{array}{l}\text { Dielectric coupling matrix of the } \\
\text { actuators }\end{array}$ \\
\hline$\xi_{d c}:$ & Closed loop average damping ratios \\
\hline$\phi_{i}:$ & $\begin{array}{l}\text { Voltage that can be applied to the } i \text { th } \\
\text { actuator }\end{array}$ \\
\hline$\phi_{\max }:$ & $\begin{array}{l}\text { Maximum voltage that can be applied to } \\
\text { the actuators }\end{array}$ \\
\hline$[M]:$ & Global mass matrix \\
\hline$[K]:$ & Global stiffness matrix \\
\hline$[C]:$ & Damping matrix \\
\hline$n:$ & Number of modes to be controlled \\
\hline$h_{p e}:$ & Thickness of piezo-patch \\
\hline$h:$ & Thickness of the plate \\
\hline$[U]:$ & $\begin{array}{l}\text { Normalized orthonormal eigenvector } \\
\text { matrix }\end{array}$ \\
\hline$\xi, \eta:$ & Local coordinates \\
\hline$n_{d}:$ & Total numbers of degrees of freedom \\
\hline$\rho:$ & Density \\
\hline$i, j, l, m:$ & Nodes numbers \\
\hline$L:$ & Length of plate \\
\hline$[N(\xi, \eta)]:$ & Shape functions \\
\hline$g=\ln \left(x_{i} /\left(x_{i+1}\right)\right):$ & Logarithmic decrement \\
\hline$N:$ & $\begin{array}{l}\text { Number of finite element of the } \\
\text { cantilever beam }\end{array}$ \\
\hline PIc: & $\begin{array}{l}\text { Performance index related to } \\
\text { controllability }\end{array}$ \\
\hline PIo: & $\begin{array}{l}\text { Performance index related to } \\
\text { observability. }\end{array}$ \\
\hline
\end{tabular}

\section{Conflict of Interests}

The authors declare that there is no conflict of interests regarding the publication of this paper.

\section{References}

[1] E. F. Crawley and J. de Luis, "Use of piezoelectric actuators as elements of intelligent structures," AIAA Journal, vol. 25, no. 10, pp. 1373-1385, 1987. 
[2] V. Gupta, M. Sharma, and N. Thakur, "Optimization criteria for optimal placement of piezoelectric sensors and actuators on a smart structure: a technical review," Journal of Intelligent Material Systems and Structures, vol. 21, no. 12, pp. 1227-1243, 2010.

[3] S. M. Yang and Y. J. Lee, "Optimization of noncollocated sensor/actuator location and feedback gain in control systems," Smart Materials and Structures, vol. 2, pp. 96-102, 1993.

[4] J. Onoda and R. T. Haftka, "An approach to structural control simultaneous optimization for large flexible spacecraft," AIAA Journal, vol. 25, no. 8, pp. 1133-1138, 1987.

[5] H. S. Tzou and H. Q. Fu, "A study of segmentation of distributed piezoelectric sensors and actuators-part I: theoretical analysis," Journal of Sound and Vibration, vol. 172, no. 2, pp. 247-259, 1994.

[6] A. Hać and L. Liu, "Sensor and actuator location in motion control of flexible structures," Journal of Sound and Vibration, vol. 167, no. 2, pp. 239-261, 1993.

[7] I. Bruant and L. Proslier, "Optimal location of actuators and sensors in active vibration control," Journal of Intelligent Material Systems and Structures, vol. 16, no. 3, pp. 197-206, 2005.

[8] S. S. Rao, T.-S. Pan, and V. B. Venkayya, "Optimal placement of actuators in actively controlled structures using genetic algorithms," AIAA Journal, vol. 29, no. 6, pp. 942-943, 1991.

[9] A. M. Sadri, J. R. Wright, and R. J. Wynne, "Modelling and optimal placement of piezoelectric actuators in isotropic plates using genetic algorithms," Smart Materials and Structures, vol. 8, no. 4, pp. 490-498, 1999.

[10] J.-H. Han and I. Lee, "Optimal placement of piezoelectric sensors and actuators for vibration control of a composite plate using genetic algorithms," Smart Materials and Structures, vol. 8, no. 2, pp. 257-267, 1999.

[11] K. R. Kumar and S. Narayanan, "The optimal location of piezoelectric actuators and sensors for vibration control of plates," Smart Materials and Structures, vol. 16, no. 6, pp. 26802691, 2007.

[12] T. Roy and D. Chakraborty, "Optimal vibration control of smart fiber reinforced composite shell structures using improved genetic algorithm," Journal of Sound and Vibration, vol. 319, no. 1-2, pp. 15-40, 2009.

[13] Y.-J. Cha, A. K. Agrawal, Y. Kim, and A. M. Raich, "Multiobjective genetic algorithms for cost-effective distributions of actuators and sensors in large structures," Expert Systems with Applications, vol. 39, no. 9, pp. 7822-7833, 2012.

[14] C. P. Smyser and K. Chandrashekhara, "Robust vibration control of composite beams using piezoelectric devices and neural networks," Smart Materials and Structures, vol. 6, no. 2, pp. 178-189, 1997.

[15] S.-H. Youn, J.-H. Han, and I. Lee, "Neuro-adaptive vibration control of composite beams subject to sudden delamination," Journal of Sound and Vibration, vol. 238, no. 2, pp. 215-231, 2000.

[16] I. Z. M. Darus and M. O. Tokhi, "Soft computing-based active vibration control of a flexible structure," Engineering Applications of Artificial Intelligence, vol. 18, no. 1, pp. 93-114, 2005.

[17] R. Kumar, S. P. Singh, and H. N. Chandrawat, "Experimental adaptive vibration control of smart structures using LVQ neural networks," Journal of Scientific and Industrial Research, vol. 65, no. 10 , pp. 798-807, 2006.

[18] A. A. Madkour, M. A. Hossain, K. P. Dahal, and H. Yu, "Intelligent learning algorithms for active vibration control,"
IEEE Transactions on Systems, Man and Cybernetics C, vol. 37, no. 5, pp. 1022-1033, 2007.

[19] L. Jinxin, C. Xuefeng, and H. E. Zhengjia, "Frequency domain active vibration control of a flexible plate based on neural networks," Frontiers of Mechanical Engineering, vol. 8, pp. 109117, 2013.

[20] J. N. Reddy, Introduction to the Finite Element Method, McGrawHill Book, New York, NY, USA, 1993.

[21] K. K. Ang, S. Y. Wang, and S. T. Quek, "Weighted energy linear quadratic regulator vibration control of piezoelectric composite plates," Smart Materials and Structures, vol. 11, no. 1, pp. 98-106, 2002.

[22] J. Li, C. Shang, and M. Zou, "Parameter optimization of linear quadratic controller based on genetic algorithm," Tsinghua Science and Technology, vol. 12, no. 1, pp. 208-211, 2007.

[23] K. Deb, Multiobjective Optimization Using Evolutionary Algorithm, John Wiley \& Sons, 2001. 

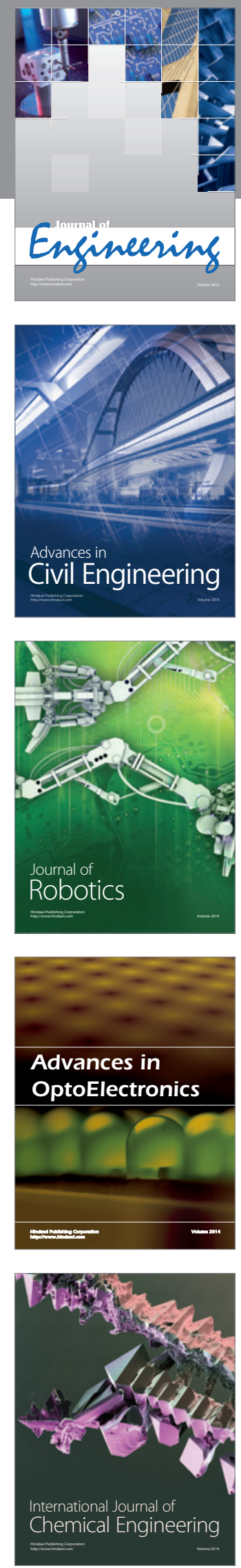

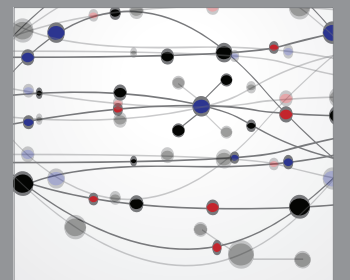

The Scientific World Journal
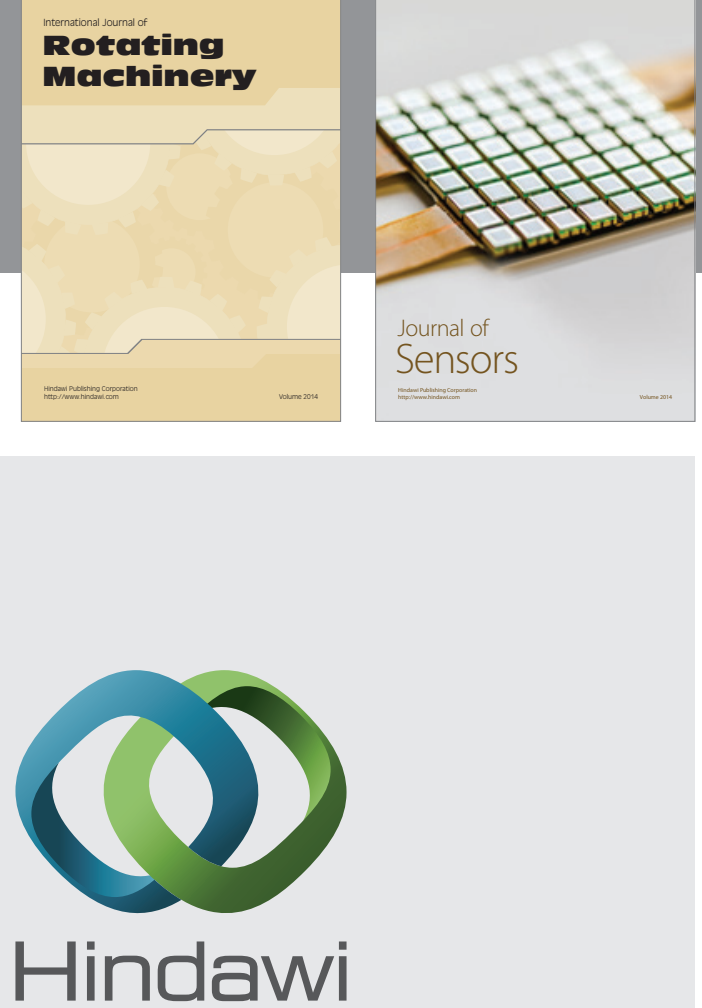

Submit your manuscripts at http://www.hindawi.com
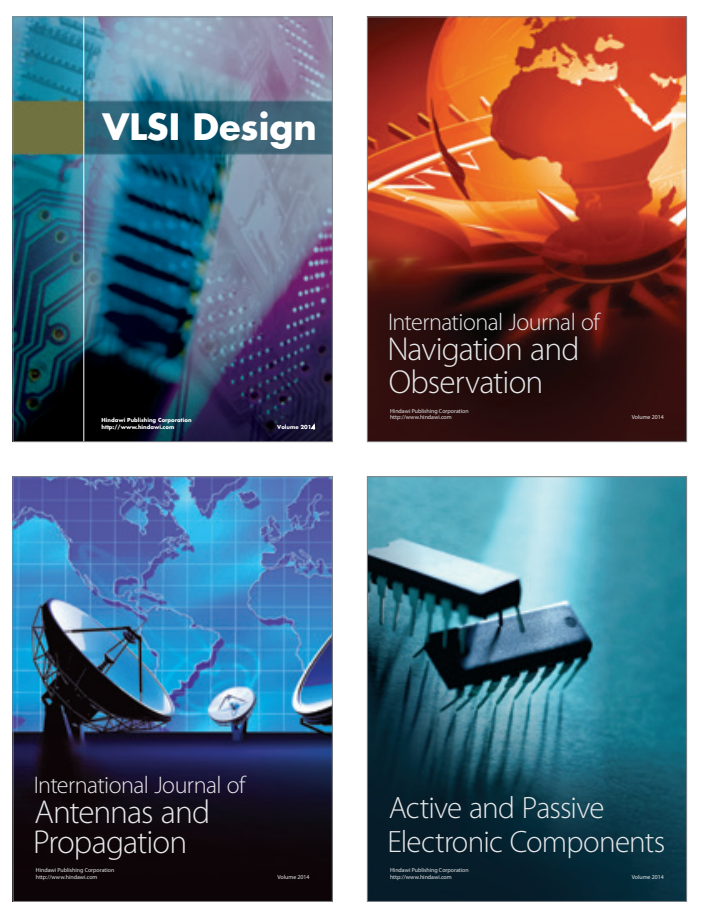
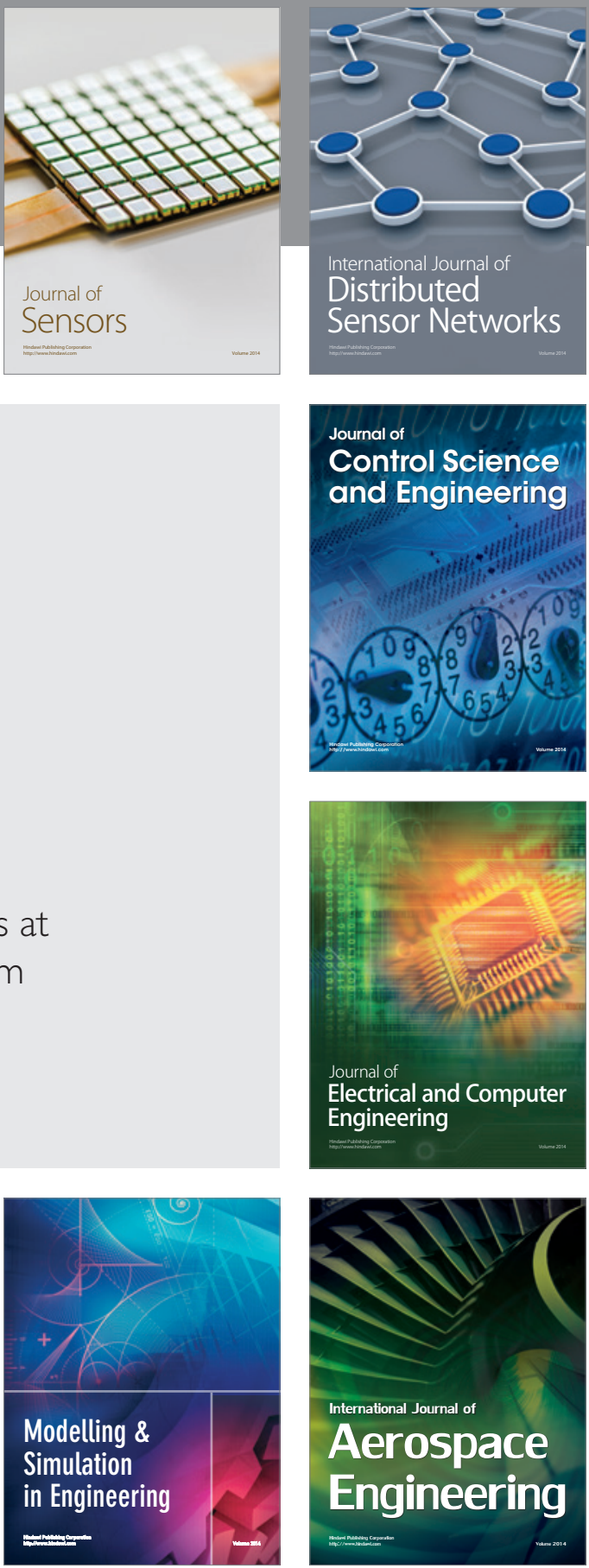

Journal of

Control Science

and Engineering
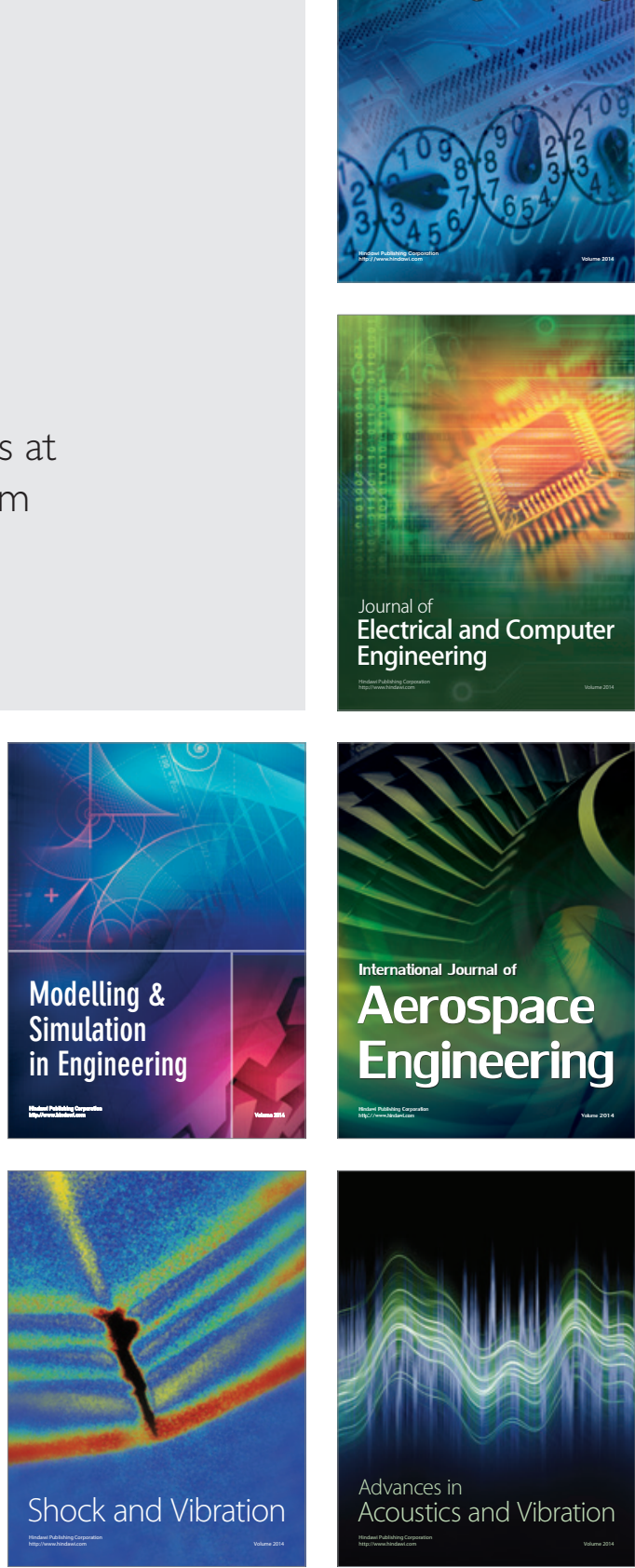